\title{
riccafd
}

Revista Iberoamericana de Ciencias de la Actividad Física y el Deporte

\section{HERRAMIENTAS TECNOLÓGICAS PARA EL ESTUDIO E INTERVENCIÓN DE LA BIOMECANICA EN EL DEPORTE DE ALTO RENDIMIENTO: UNA MIRADA DESDE FISIOTERAPIA}

TECHNOLOGICAL TOOLS FOR THE STUDY AND INTERVENTION OF BIOMECHANICS IN HIGH PERFORMANCE SPORT: A VIEW FROM PHYSIOTHERAPY

Alfonso Mantilla José Iván.

Fisioterapeuta, Universidad del Rosario, Colombia. Josealfonso25@hotmail.com

Correspondencia: José Iván Alfonso Mantilla. josealfonso25@hotmail.com

Código UNESCO: 321311. Fisioterapia

Clasificación Consejo de Europa: 14. Fisioterapia y Rehabilitación

Recibido el 10 de marzo de 2019

Aceptado el 23 de julio de 2019

DOI: http://dx.doi.org/10.24310/10.24310/riccafd.2019.v8i3.7491

\section{RESUMEN}

La biomecánica es el área de estudio de componentes cinéticos y cinemáticos implicados en el movimiento corporal humano profundizando en elementos tales como fuerza muscular, rangos de movimiento, aceleración, velocidad y desplazamiento. En el deporte de alto rendimiento, el estudio de las variables biomecánicas que inciden en el gesto motor es de vital importancia para entender los mecanismos de control y adaptación del movimiento a actividades especificas, con el fin de desarrollar programas para mejorar la ejecución del movimiento en determinadas fases del gesto deportivo y prevenir lesiones a partir del estudio del gesto motor. Por tal motivo el objetivo de este artículo es realizar una revisión sistemática de la literatura sobre el uso de la tecnología para la evaluación e intervención de la biomecánica en el deporte de alto rendimiento.

palabras clave: Rehabilitación, tecnología, medicina deportiva, alto rendimiento, biomecánica.

\section{ABSTRACT}

Biomechanics is the area of study of kinematic and kinematic components involved in human body movement by delving into elements such as muscle strength, ranges of motion, acceleration, velocity and displacement. In high performance sport, the study of the biomechanical variables that affect the motor gesture is of vital importance to understand the mechanisms of control and adaptation of the 
movement to specific activities, in order to develop programs to improve the execution of the movement in certain phases of the sports gesture and prevent injuries from the study of the motor gesture. For this reason the aim of this article is to carry out a systematic review of the literature on the use of technology for the evaluation and intervention of biomechanics in high performance sport.

key words: Rehabilitation, technology, sports medicine, athletic performance, biomechanics.

\section{INTRODUCCIÓN}

La fisioterapia es el área de la salud encargada del estudio del movimiento corporal humano, esta estudia las relaciones patológicas que pueden llevar a una lesión de los sistemas cardiovascular, neuromuscular y osteomuscular(1). El movimiento corporal humano, se traduce en un conjunto de elementos que permiten el desarrollo de actividades de la vida diaria en distintos roles del ser humano. De hecho, la biomecánica es el área de estudio de componentes cinéticos y cinemáticos implicados en el movimiento corporal humano profundizando en elementos tales como fuerza muscular, rangos de movimiento, aceleración, velocidad y desplazamiento(2, 3). En el deporte de alto rendimiento, el estudio de las variables biomecánicas que inciden en el gesto motor es de vital importancia para entender los mecanismos de control y adaptación del movimiento a actividades especificas, con el fin de desarrollar programas para mejorar la ejecución del movimiento en determinadas fases del gesto deportivo, prevenir lesiones a partir del estudio del gesto motor, examinar déficit musculares y disminución en el rango de movimiento que puedan intervenir en determinada acción específica del gesto deportivo (4-9).

El deporte de alto rendimiento siempre ha inquietado a diferentes investigadores de todo el mundo debido a que diversos deportistas rompen records en diferentes disciplinas deportivas en competiciones internacionales. Esto ha sido motivo de análisis por diferentes entidades de investigación del deporte(10-13). La biomecánica, es un área que facilita el estudio de variables específicas del movimiento corporal humano que pueden dar conclusiones en relación a las capacidades físicas de deportistas en distintas disciplinas. Por ejemplo, estudios biomecanicos pueden determinar características como aceleración, velocidad, disipación y conservación de energía, resistencia aerodinámica, fuerzas de fricción, fuerzas de reacción, giros, torque, centro de masa y centro de gravedad. Estas características, permiten determinar acciones que se deben tomar para mejorar el rendimiento del deportista, prevenir lesiones y aumentar cualidades físicas que permitan mejorar su rendimiento en competencias y la obtención de logros deportivos(14).De hecho, estudios biomecanicos han demostrado mejorar el aprendizaje y control motor de deportistas aumentando sus cualidades físicas y mejorando la ejecución de tareas especificas que permiten que el gesto motor se realice con una mayor eficacia aumentando las probabilidades de éxito en competencias(15-17). En la actualidad, el estudio del movimiento corporal humano en deportistas se realiza con la ayuda de tecnología debido a que permite hacer monitoreo digitales de variables que pueden ser cuantificables y conver- 
tirse en indicadores de seguimiento en el deportista(18). Por tal motivo, el objetivo de este estudio es realizar una revisión sistemática de la literatura sobre el uso de la tecnología para la evaluación e intervención de la biomecánica en el deporte de alto rendimiento.

\section{MATERIAL Y METODOS}

Se realizo una revisión sistemática de la literatura. Se determinaron los siguientes criterios de inclusión y exclusión:

Inclusión: Estudios contemplados del año 2000 al 2017, literatura que contemplara los siguientes términos MeSH: Rehabilitation, technology, sports medicine, athletic performance, biomechanics. La literatura se aceptaría en idioma español, ingles y portugués

Se estableció como otro criterio de inclusión que la búsqueda de evidencia se basara en: Fuentes primarias: Estudios ECA, Metaanálisis, casos y controles, estudios de cohorte, revisiones sistemáticas, revisiones de literatura. Fuentes secundarias: Monografías, tesis de grado, libros.

Exclusión: Estudios que su año de publicación fuera inferior al año 2000, que no contemplaran los términos MeSH establecidos, estudios que no estuvieran disponibles o completos

\section{RESULTADOS}

En la figura 1 se muestra el proceso de extracción de la evidencia identificada en las bases de datos y su proceso de selección

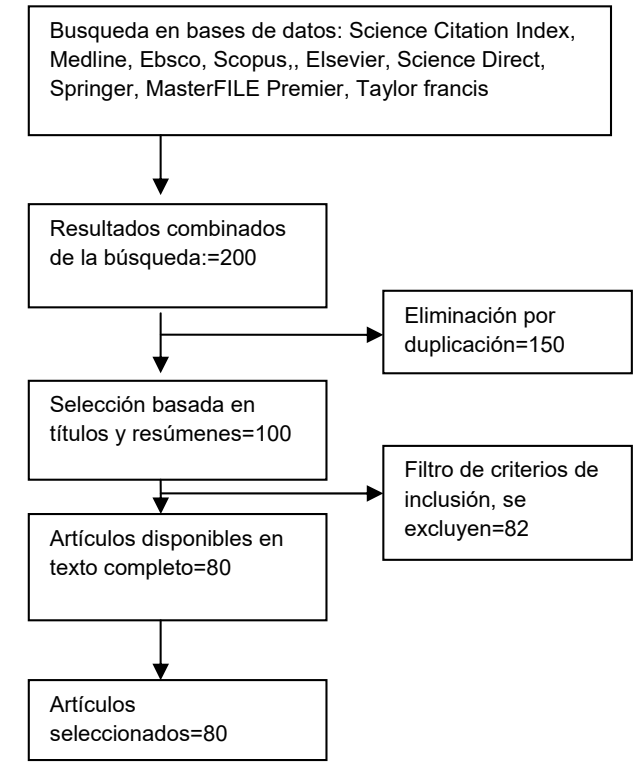

Fuente de elaboración propia 2016

Figura 1 (Flujograma de extracción de la evidencia).

\begin{tabular}{l|l}
\hline 69 & HERRAMIENTAS TECNOLÓGICAS PARA EL ESTUDIO E INTERVENCIÓN \\
& DE LA BIOMECANICA EN EL DEPORTE DE ALTO RENDIMIENTO: \\
UNA MIRADA DESDE FISIOTERAPIA
\end{tabular}




\section{Fuente de elaboración propia 2016}

Dentro de la evidencia se pudo encontrar información en relación al uso de tecnología en el análisis de la biomecánica del movimiento corporal humano en deportistas de alto rendimiento que cuentan con criterios de validez y confiabilidad.

\section{Análisis biomecanico a través de sistemas de captura de movimiento y cámaras profundas}

Unas de las herramientas utilizadas en el deporte de alto rendimiento son las cámaras con sistema de captura a través de marcadores de movimiento que pueden escanear el gesto motor y dar resultados objetivos sobre ángulos, fuerza, rango de movimiento, rotación, magnitud de torque que pueden determinar información sobre características especificas del gesto motor y riesgo de lesión por factores como masa muscular y posición de segmentos corporales. Lo anterior, puede ser usado para mejorar el rendimiento del deportista utilizando como indicadores de seguimiento los elementos de evaluación de la biomecánica corporal(19-23). Además, se reporta el uso de cámaras profundas para el análisis de marcha como un elemento esencial en la evaluación fisioterapéutica, se utilizan sistemas de cámaras profundas para reconstruir el movimiento en 3D durante la marcha para detectar alteraciones que pueden ser generadas por patologías 0 alteraciones musculares que afecten la movilidad normal $(24,25)$.

\section{Plataforma de fuerza y Baropodometría}

Se pueden realizar evaluaciones de la fuerza ejercida en actividades dinámicas o estáticas con la ayuda de dispositivos que cuantifican la cantidad de fuerza aplicada en una actividad específica lo cual puede ayudar a realizar análisis profundos de bases musculares implicadas en el gesto motor del deportista. Además, se pueden hacer evaluaciones de la tensión muscular que ocasionan alteraciones posturales, se pueden hacer mediciones de fases de velocidad y calcular la variabilidad y simetría en deportistas que puede determinar asimetrías musculares que afectan el gesto motor, rendimiento deportivo y aumentar el riesgo de lesión. Para ejemplificar, se realizan evaluaciones de la influencia de parámetros biomecanicos en salto que pueden dar información en relación a simetrías musculares, movilidad articular y picos de fuerza. Esto, puede ayudar a correlacionar el gesto motor con la activación muscular donde se pueden crear intervenciones de perfeccionamiento para mejorar asimetrías musculares y posturales(26-33). De igual manera, se ha reportado la importancia del estudio del centro de masa y centro de gravedad en la postura como base de la estabilización articular y muscular, las plataformas de presión permiten realizar la mediciones de presión y desplazamiento con la cual se puede realizar diagnósticos de disfunciones de cadenas musculares implicadas en gestos motores específicos(34-37).

\section{Electromiografía}

Este es uno de los metodos utilizados a nivel mundial para verificar la actividad eléctrica de la activación muscular, este se realiza a través de sensores con 
electrodos activos que detectan una señal eléctrica y permiten realizar análisis estáticos y dinámicos. En deporte, el uso de la electromiografía permite realizar evaluaciones comparativas de picos de activación muscular para determinar relaciones con gesto motor y entrenamiento muscular. Por ejemplo, se puede identificar actividad anormal de la musculatura antagonista que afecta el rendimiento deportivo aumentando la demanda metabólica y disminuyendo la estabilidad articular. Además, el aumento de actividad muscular antagonista puede ocasionar aumento entre las superficies articulares ocasionado asimetrías musculares. Gracias a este análisis puede hacerse intervenciones precisas con el fin de corregir asimetrías musculares para prevenir lesiones, Mejorar el gesto motor, aumento de las cualidades físicas y habilidades en el campo de juego(38-44).

\section{Acelerómetros y giroscopios}

Otra herramienta utilizada en alto rendimiento son los acelerómetros, los cuales son dispositivos que miden la fuerza generada a través de vibraciones en el cambio de movimiento en actividades dinámicas. En el deporte, se puede hacer mediciones de parámetros como velocidad de ciclo, desplazamiento, aceleración, toque, energía potencial para crear estrategias en prevención de lesiones a partir de estos análisis biomecanicos específicos. Adicionalmente, se pueden utilizar estos dispositivos para la mediciones de aceleración rotacional, velocidad, picos de velocidad rotacional durante el impacto en la cabeza que producen contusiones intracraneales que pueden ocasionar lesiones cerebrales en diferentes deportes. Adicionalmente, los acelerómetros son efectivos en la evaluación de estabilidad postural y el desplazamiento del centro de masa y gravedad con el fin de determinar alteraciones que puedan afectar el gesto deportivo. Para finalizar, estos también pueden ser usados para la medición del gasto metabólico durante la actividad física realizada lo cual ayuda en la estandarización de programas de prescripción de ejercicio en jugadores de alto rendimiento(45-59).

\section{Software electrónicos}

En la actualidad, la tecnología y el razonamiento clínico se combinan para crear diagnósticos e intervenciones con mejores resultados. Este es el caso del los software de estudio de marcha para la determinación de características cinéticas y cinemáticas de la marcha en deportistas, adultos sanos o adultos con patología. Entre los software más conocidos y validados se encuentran sistema Opto Gait, sistema BTS y Kinovea; estos permiten tener un control del movimiento del paciente y realizar una medición de forma cuantitativa de parámetros específicos de la marcha como longitud de paso, longitud de zancada, distancia, tiempo de contacto total, tiempo de paso, velocidad de marcha , aceleración, tiempo de paso progresivo, cadencia, ciclo de marcha, fase de apoyo, fase de balanceo, fase de contacto con el talón, fase de pie plano, fase de despegue, soporte donde se puede evaluar condiciones físicas, identificación de deficiencias y asimetrías musculares y ángulos de movimiento. Gracias a esto, se generan programas de rehabilitación específicos con resultados cuantificables, se controla de forma periódica el proceso de rehabilitación y se crean bases estadísticas para realizar estudios de normalización de datos(60-71). 


\section{Sistemas de evaluación isocinetica}

La fuerza es un elemento central de evaluación en deportistas de alto rendimiento, una de las maquinas utilizadas para la evaluación de la fuerza isocinetica, excéntrica y concéntrica es el sistema HUMAC el cual permite realizar procesos de evaluación, intervención y entrenamiento de la fuerza. Este sistema proporciona información en relación a picos de torque, trabajo total, relación agonista y antagonista de musculatura de miembros inferiores o superiores y permite determinar diagnósticos más precisos y tener datos cuantificables para el desarrollo de programas específicos para el deportista(72-74).

\section{Sistemas de entrenamiento y rehabilitación Aretech Zerog, ZeroG overground y Alter $\mathbf{G}$}

En la actualidad, la rehabilitación ha desarrollado nuevos sistemas de intervención para garantizar mejores procesos, se han creado sistemas de soporte de peso para rehabilitación de marcha después de lesiones basados en efectos anti gravitatorios para facilitar la adopción del gesto motor, estos dispositivos han sido desarrollados en base a bandas sin fin adaptadas para realizar procesos de rehabilitación con mejores resultados. Esto ocurre debido a que se reduce la carga de las extremidades inferiores y se puede variar la cantidad de presión para realizar procesos de rehabilitación controlados en base al porcentaje de soporte de los tejidos musculoesqueléticos durante las fases de rehabilitación(75-80).

\section{DISCUSIÓN}

Los análisis biomecanicos son un pilar fundamental en el estudio del movimiento corporal humano, con ayuda de estos análisis se pueden identificar alteraciones en el gesto motor, desempeño muscular, rango de movimiento, flexibilidad y función motora en deportistas de alto rendimiento. Con la realización de análisis biomecanicos precisos, se puede realizar una estandarización de poblaciones de deportistas para identificar posibles alteraciones que pueden estar relacionadas con lesiones musculares. Es por eso, que los análisis biomecanicos deben realizarse de manera profunda utilizando la tecnología como aliada para tener resultados cuantificables y medibles. En el deporte de alto rendimiento, realizar análisis biomecanicos a los deportistas permiten establecer criterios para prescribir programas de prevención de lesiones, potenciación de capacidades físicas, corrección de gesto motor, reducir el gasto metabólico durante el ejercicio lo cual puede traer resultados satisfactorios y aumentar la probabilidad de obtener títulos a nivel suramericano, panamericano y olímpico. Estos análisis deben ser realizados por profesionales que comprendan los mecanismos del cuerpo humano a nivel biomecanico, muscular y articular con el fin de que combinen el uso de la tecnología con el razonamiento clínico para determinar objetivos específicos en los ciclos de preparación de deportistas de alto rendimiento. En fisioterapia, es necesario realizar estudios con diferentes tipos de población con el fin de determinar parámetros de normalidad y crear protocolos de intervención y evaluación estandarizados para usar la tecnología 
como medio de crecimiento profesional de la fisioterapia a nivel nacional e internacional. Adicionalmente, la tecnología es la base para el avance global en áreas de la salud. Por eso es que los profesionales en fisioterapia deben ser capaces de entender que estas herramientas ayudan a determinar una mejor evaluación e intervención con seguimiento de indicadores cuantificables para prestar una mejor atención al deportista. De hecho, el uso de la tecnología en el deporte se ha relacionado con el deporte olímpico con el uso de análisis biomecanicos para mejorar procesos de adquisición de habilidades y corrección de gesto motor para competiciones. En contraste, a nivel paralímpico la tecnología es usada por los fisioterapeutas para la creación y adaptación de prótesis y aparatos adaptativos para los deportistas y sus constantes mejoras en diseño y mecanismos tecnológicos para el deporte.

\section{LIMITACIONES Y CAMINOS FUTUROS}

En Colombia, el acceso a tecnología para procesos de rehabilitación ha aumentado debido a la adquisición de equipos tecnológicos por parte de entidades prestadoras de servicio de salud especializadas en fisioterapia. A nivel educativo, las universidades a nivel nacional entienden la importancia de la enseñanza a través de tecnología con el fin de que los estudiantes estén capacitados para el manejo de diferentes equipos. La educación en fisioterapia debe tener como objetivo acoplar la tecnología para sus procesos de rehabilitación con el fin de obtener indicadores cuantificables en rehabilitación que puedan ser usados para realizar investigaciones con diferentes tipos de población.

\section{CONCLUSIONES}

En Colombia, el acceso a tecnología para procesos de rehabilitación ha aumentado debido a la adquisición de equipos tecnológicos por parte de entidades prestadoras de servicio de salud especializadas en fisioterapia. A nivel educativo, las universidades a nivel nacional entienden la importancia de la enseñanza a través de tecnología con el fin de que los estudiantes estén capacitados para el manejo de diferentes equipos. La educación en fisioterapia debe tener como objetivo acoplar la tecnología para sus procesos de rehabilitación con el fin de obtener indicadores cuantificables en rehabilitación que puedan ser usados para realizar investigaciones con diferentes tipos de población.

\section{CONFLICTO DE INTERESES}

Los autores declaran que no existe conflicto de intereses.

\section{BIBLIOGRAFIA}

1. Lesmes JD. Evaluación clínico-funcional del movimiento corporal humano: Ed. Médica Panamericana; 2007. 
2. José A. Biomecánica deportiva y control del entrenamiento: Funámbulos Editores; 2009.

3. Izquierdo M, Redín MI. Biomecánica y bases neuromusculares de la actividad física y el deporte: Ed. Médica Panamericana; 2008.

4. Nicola TL, Jewison DJ. The anatomy and biomechanics of running. Clin Sports Med. 2012;31(2):187-201.

5. Pappas E, Nightingale EJ, Simic M, Ford KR, Hewett TE, Myer GD. Do exercises used in injury prevention programmes modify cutting task biomechanics? A systematic review with meta-analysis. Br J Sports Med. 2015;49(10):673-80.

6. Weiss K, Whatman C. Biomechanics Associated with Patellofemoral Pain and ACL Injuries in Sports. Sports Med. 2015;45(9):1325-37.

7. Thompson JA, Tran AA, Gatewood CT, Shultz R, Silder A, Delp SL, et al. Biomechanical Effects of an Injury Prevention Program in Preadolescent Female Soccer Athletes. Am J Sports Med. 2017;45(2):294-301.

8. Dugan SA, Bhat KP. Biomechanics and analysis of running gait. Phys Med Rehabil Clin N Am. 2005;16(3):603-21.

9. Chow JW, Knudson DV. Use of deterministic models in sports and exercise biomechanics research. Sports Biomech. 2011;10(3):219-33.

10. Sanchis-Gomar F, Pareja-Galeano H, Rodriguez-Marroyo JA, de Koning JJ, Lucia A, Foster C. Olympic Genes on the Podium? Int J Sports Physiol Perform. 2016;11(7):973-4.

11. Allen SV, Vandenbogaerde TJ, Pyne DB, Hopkins WG. Predicting a nation's olympicqualifying swimmers. Int J Sports Physiol Perform. 2015;10(4):431-5.

12. Lucia A, Moran $M$, Zihong $H$, Ruiz JR. Elite athletes: are the genes the champions? Int J Sports Physiol Perform. 2010;5(1):98-102.

13. Mantilla JIA, Santa JM, Vargas OC. KINESIOGENÓMICA: UNA NUEVA PERSPECTIVA DE INVESTIGACIÓN EN FISIOTERAPIA. Revista Movimiento Científico. 2016;10(1):78-86.

14. Hebert-Losier K, Supej M, Holmberg HC. Biomechanical factors influencing the performance of elite Alpine ski racers. Sports Med. 2014;44(4):519-33.

15. Keogh JW, Hume PA. Evidence for biomechanics and motor learning research improving golf performance. Sports Biomech. 2012;11(2):288-309.

16. Ferreira MI, Barbosa TM, Costa MJ, Neiva HP, Marinho DA. Energetics, Biomechanics, and Performance in Masters' Swimmers: A Systematic Review. J Strength Cond Res. 2016;30(7):2069-81.

17. Keogh JW. Paralympic sport: an emerging area for research and consultancy in sports biomechanics. Sports Biomech. 2011;10(3):234-53.

18. Verhagen E, Bolling C. Protecting the health of the @hlete: how online technology may aid our common goal to prevent injury and illness in sport. $\mathrm{Br} \mathrm{J}$ Sports Med. 2015;49(18):1174-8.

19. Abrams GD, Harris AH, Andriacchi TP, Safran MR. Biomechanical analysis of three tennis serve types using a markerless system. Br J Sports Med. 2014;48(4):339-42.

20. Sheets AL, Abrams GD, Corazza S, Safran MR, Andriacchi TP. Kinematics differ- 
ences between the flat, kick, and slice serves measured using a markerless motion capture method. Ann Biomed Eng. 2011;39(12):3011-20.

21. Corazza S, Mundermann L, Chaudhari AM, Demattio T, Cobelli C, Andriacchi TP. A markerless motion capture system to study musculoskeletal biomechanics: visual hull and simulated annealing approach. Ann Biomed Eng. 2006;34(6):1019-29.

22. Sandau M, Koblauch H, Moeslund TB, Aanaes H, Alkjaer T, Simonsen EB. Markerless motion capture can provide reliable $3 \mathrm{D}$ gait kinematics in the sagittal and frontal plane. Med Eng Phys. 2014;36(9):1168-75.

23. Abrams GD, Sheets AL, Andriacchi TP, Safran MR. Review of tennis serve motion analysis and the biomechanics of three serve types with implications for injury. Sports Biomech. 2011;10(4):378-90.

24. Auvinet E, Multon F, Meunier J. Gait analysis with multiple depth cameras. Conf Proc IEEE Eng Med Biol Soc. 2011;2011:6265-8.

25. Kwon $\mathrm{YH}$, Casebolt JB. Effects of light refraction on the accuracy of camera calibration and reconstruction in underwater motion analysis. Sports Biomech. 2006;5(1):95-120.

26. Korhonen MT, Suominen H, Viitasalo JT, Liikavainio T, Alen M, Mero AA. Variability and symmetry of force platform variables in maximum-speed running in young and older athletes. J Appl Biomech. 2010;26(3):357-66.

27. Pastorelli F, Pasquetti P. Biomechanical analysis and rehabilitation in athletes. Clin Cases Miner Bone Metab. 2013;10(2):96.

28. Pietraszewski B, Struzik A. Evaluation of selected biomechanical parameters in female team sports players. Acta Bioeng Biomech. 2013;15(4):103-8.

29. Sinitski EH, Lemaire ED, Baddour N. Evaluation of motion platform embedded with dual belt treadmill instrumented with two force plates. J Rehabil Res Dev. 2015;52(2):221-34.

30. Plessa EI, Rousanoglou EN, Boudolos KD. Comparison of the take-off ground reaction force patterns of the pole vault and the long jump. J Sports Med Phys Fitness. 2010;50(4):416-21.

31. Fritz M, Peikenkamp K. Simulation of the influence of sports surfaces on vertical ground reaction forces during landing. Med Biol Eng Comput. 2003;41(1):11-7.

32. Ericksen HM, Gribble PA, Pfile KR, Pietrosimone BG. Different modes of feedback and peak vertical ground reaction force during jump landing: a systematic review. J Athl Train. 2013;48(5):685-95.

33. Liu H, Wu W, Yao W, Spang JT, Creighton RA, Garrett WE, et al. Effects of knee extension constraint training on knee flexion angle and peak impact ground-reaction force. Am J Sports Med. 2014;42(4):979-86.

34. Khorievin VI, Horkovenko AV, Vereshchaka IV. [Displacement of center of pressure on the support and changes of the joint angles of the lower extremity at squatting]. Fiziol Zh. 2012;58(3):32-42.

35. Mullin DS, King GW, Saripalle SK, Derakhshani RR, Lovelace CT, Burgoon JK. Deception effects on standing center of pressure. Hum Mov Sci. 2014;38:106-15.

36. Mann R, Malisoux L, Urhausen A, Meijer K, Theisen D. Plantar pressure measurements and running-related injury: A systematic review of methods and possible associations. Gait Posture. 2016;47:1-9. 
37. Cretual A. Which biomechanical models are currently used in standing posture analysis? Neurophysiol Clin. 2015;45(4-5):285-95.

38. MacKenzie SJ, Lavers RJ, Wallace BB. A biomechanical comparison of the vertical jump, power clean, and jump squat. J Sports Sci. 2014;32(16):1576-85.

39. Tartaruga MP, Brisswalter J, Peyre-Tartaruga LA, Avila AO, Alberton CL, Coertjens $\mathrm{M}$, et al. The relationship between running economy and biomechanical variables in distance runners. Res Q Exerc Sport. 2012;83(3):367-75.

40. Knarr BA, Zeni JA, Jr., Higginson JS. Comparison of electromyography and joint moment as indicators of co-contraction. J Electromyogr Kinesiol. 2012;22(4):607-11.

41. Lloyd DG, Besier TF. An EMG-driven musculoskeletal model to estimate muscle forces and knee joint moments in vivo. J Biomech. 2003;36(6):765-76.

42. Clarys JP. Electromyography in sports and occupational settings: an update of its limits and possibilities. Ergonomics. 2000;43(10):1750-62.

43. Howard RM, Conway R, Harrison AJ. A survey of sensor devices: use in sports biomechanics. Sports Biomech. 2016;15(4):450-61.

44. Escamilla RF, Andrews JR. Shoulder muscle recruitment patterns and related biomechanics during upper extremity sports. Sports Med. 2009;39(7):569-90.

45. He B, Bai J, Zipunnikov VV, Koster A, Caserotti P, Lange-Maia B, et al. Predicting human movement with multiple accelerometers using movelets. Med Sci Sports Exerc. 2014;46(9):1859-66.

46. Xiao L, He B, Koster A, Caserotti P, Lange-Maia B, Glynn NW, et al. Movement prediction using accelerometers in a human population. Biometrics. 2016;72(2):513-24.

47. Myklebust $\mathrm{H}$, Losnegard T, Hallen J. Differences in V1 and V2 ski skating techniques described by accelerometers. Scand J Med Sci Sports. 2014;24(6):882-93.

48. Broglio SP, Surma T, Ashton-Miller JA. High school and collegiate football athlete concussions: a biomechanical review. Ann Biomed Eng. 2012;40(1):37-46.

49. Bradshaw EJ, Hume PA. Biomechanical approaches to identify and quantify injury mechanisms and risk factors in women's artistic gymnastics. Sports Biomech. 2012;11(3):324-41.

50. Allison MA, Kang YS, Maltese MR, Bolte JHt, Arbogast KB. Measurement of Hybrid III Head Impact Kinematics Using an Accelerometer and Gyroscope System in Ice Hockey Helmets. Ann Biomed Eng. 2015;43(8):1896-906.

51. Allison MA, Kang YS, Bolte JHt, Maltese MR, Arbogast KB. Validation of a helmetbased system to measure head impact biomechanics in ice hockey. Med Sci Sports Exerc. 2014;46(1):115-23.

52. Campbell KR, Warnica MJ, Levine IC, Brooks JS, Laing AC, Burkhart TA, et al. Laboratory Evaluation of the gForce Tracker, a Head Impact Kinematic Measuring Device for Use in Football Helmets. Ann Biomed Eng. 2016;44(4):1246-56.

53. Alberts JL, Hirsch JR, Koop MM, Schindler DD, Kana DE, Linder SM, et al. Using Accelerometer and Gyroscopic Measures to Quantify Postural Stability. J Athl Train. 2015;50(6):578-88.

54. Ma J, Kharboutly $H$, Benali A, Benamar F, Bouzit M. Joint angle estimation with accelerometers for dynamic postural analysis. J Biomech. 2015;48(13):3616-24. 
55. Herman Hansen B, Bortnes I, Hildebrand M, Holme I, Kolle E, Anderssen SA. Validity of the ActiGraph GT1M during walking and cycling. J Sports Sci. 2014;32(6):510-6.

56. King GA, Torres N, Potter C, Brooks TJ, Coleman KJ. Comparison of activity monitors to estimate energy cost of treadmill exercise. Med Sci Sports Exerc. 2004;36(7):1244-51.

57. Dong B, Biswas S, Montoye A, Pfeiffer K. Comparing metabolic energy expenditure estimation using wearable multi-sensor network and single accelerometer. Conf Proc IEEE Eng Med Biol Soc. 2013;2013:2866-9.

58. Nguyen DM, Lecoultre V, Sunami Y, Schutz Y. Assessment of physical activity and energy expenditure by GPS combined with accelerometry in real-life conditions. J Phys Act Health. 2013;10(6):880-8.

59. Alfonso Mantilla J. USOS DE LOS ACELERÓMETROS EN FISIOTERAPIA: UNA REVISIÓN DE LA LITERATURA. Revista Iberoamericana de Ciencias de la Actividad Física y el Deporte. 2017;6(2).

60. Wahl Y, Duking P, Droszez A, Wahl P, Mester J. Criterion-Validity of Commercially Available Physical Activity Tracker to Estimate Step Count, Covered Distance and Energy Expenditure during Sports Conditions. Front Physiol. 2017;8:725.

61. Roche-Seruendo LE, Garcia-Pinillos F, Haicaguerre J, Bataller-Cervero AV, SotoHermoso VM, Latorre-Roman PA. Lack of influence of muscular performance parameters on spatio-temporal adaptations with increased running velocity. J Strength Cond Res. 2017.

62. Lienhard K, Schneider D, Maffiuletti NA. Validity of the Optogait photoelectric system for the assessment of spatiotemporal gait parameters. Med Eng Phys. 2013;35(4):500-4.

63. Gomez Bernal A, Becerro-de-Bengoa-Vallejo R, Losa-Iglesias ME. Reliability of the OptoGait portable photoelectric cell system for the quantification of spatial-temporal parameters of gait in young adults. Gait Posture. 2016;50:196-200.

64. Cho M, Jun I. Effects of pelvic adjustment on female university students' gait variables. J Phys Ther Sci. 2014;26(5):759-62.

65. Engelson MA, Bruns R, Nightingale CJ, Bardwell KM, Mason CA, Tu S, et al. Validation of the OptoGait System for Monitoring Treatment and Recovery of Post-Concussion Athletes. J Chiropr Med. 2017;16(2):163-9.

66. Kyrarini $M$, Wang $X$, Gräser A, editors. Comparison of vision-based and sensorbased systems for joint angle gait analysis. Medical Measurements and Applications (MeMeA), 2015 IEEE International Symposium on; 2015: IEEE.

67. Sayeed T, Samà A, Català A, Cabestany J, editors. Comparison and adaptation of step length and gait speed estimators from single belt worn accelerometer positioned on lateral side of the body. Intelligent Signal Processing (WISP), 2013 IEEE 8th International Symposium on; 2013: IEEE.

68. Breen S, Stephenson ML, Jensen R, Drum S, editors. Changes in running gait parameters during a $161 \mathrm{~km}$ trail race. ISBS-Conference Proceedings Archive; 2014.

69. Mackala K, Stodolka J, Siemienski A, Coh M. Biomechanical analysis of squat jump and countermovement jump from varying starting positions. J Strength Cond Res. 2013;27(10):2650-61.

70. Dworak LB, Murawa M, Owsian M, Maczynski J, Kabacinski J, Rzepnicka A. Three point crutch gait from the perspective of biomechanics and kinesiology. State of knowl- 
edge and idea behind the research. Chir Narzadow Ruchu Ortop Pol. 2011;76(5):30512, 278-85.

71. Struzik A, Pietraszewski B, Zawadzki J. Biomechanical analysis of the jump shot in basketball. J Hum Kinet. 2014;42:73-9.

72. Ardern CL, Pizzari T, Wollin MR, Webster KE. Hamstrings strength imbalance in professional football (soccer) players in Australia. J Strength Cond Res. 2015;29(4):9971002.

73. de Araujo Ribeiro Alvares JB, Rodrigues R, de Azevedo Franke R, da Silva BG, Pinto RS, Vaz MA, et al. Inter-machine reliability of the Biodex and Cybex isokinetic dynamometers for knee flexor/extensor isometric, concentric and eccentric tests. Phys Ther Sport. 2015;16(1):59-65.

74. Impellizzeri FM, Bizzini M, Rampinini E, Cereda F, Maffiuletti NA. Reliability of isokinetic strength imbalance ratios measured using the Cybex NORM dynamometer. Clin Physiol Funct Imaging. 2008;28(2):113-9.

75. Hidler J, Brennan D, Black I, Nichols D, Brady K, Nef T. ZeroG: overground gait and balance training system. J Rehabil Res Dev. 2011;48(4):287-98.

76. Fenuta AM, Hicks AL. Muscle activation during body weight-supported locomotion while using the ZeroG. J Rehabil Res Dev. 2014;51(1):51-8.

77. Mikami Y, Fukuhara K, Kawae T, Kimura H, Ochi M. The effect of anti-gravity treadmill training for prosthetic rehabilitation of a case with below-knee amputation. Prosthet Orthot Int. 2015;39(6):502-6.

78. Bugbee WD, Pulido PA, Goldberg T, D'Lima DD. Use of an Anti-Gravity Treadmill for Early Postoperative Rehabilitation After Total Knee Replacement: A Pilot Study to Determine Safety and Feasibility. Am J Orthop (Belle Mead NJ). 2016;45(4):E167-73.

79. McNeill DK, de Heer HD, Bounds RG, Coast JR. Accuracy of unloading with the antigravity treadmill. J Strength Cond Res. 2015;29(3):863-8.

80. Saxena A, Granot A. Use of an anti-gravity treadmill in the rehabilitation of the operated achilles tendon: a pilot study. J Foot Ankle Surg. 2011;50(5):558-61.

Referencias totales citadas: 80

Referencias citadas correspondientes a la Rev Ib CC Act Fis Dep: 1 